\title{
HYPERIDEAL CIRCLE PATTERNS
}

\author{
JEAN-MARC SCHLENKER
}

\begin{abstract}
A "hyperideal circle pattern" in $S^{2}$ is a finite family of oriented circles, similar to an "usual" circle pattern but such that the closed disks bounded by the circles do not cover the whole sphere. Hyperideal circle patterns are directly related to hyperideal hyperbolic polyhedra, and also to circle packings.

To each hyperideal circle pattern, one can associate an incidence graph and a set of intersection angles. We characterize the possible incidence graphs and intersection angles of hyperideal circle patterns in the sphere, the torus, and in higher genus surfaces. It is a consequence of a more general result, describing the hyperideal circle patterns in the boundaries of geometrically finite hyperbolic 3-manifolds (for the corresponding $\mathbb{C} P^{1}$-structures). This more general statement is obtained as a consequence of a theorem of Otal [Ota94, BO01] on the pleating laminations of the convex cores of geometrically finite hyperbolic manifolds.
\end{abstract}

\section{Résumé}

Un "motif de cercles hyperidéal" sur $S^{2}$ est une famille finie de cercles orientés, similaire à un motif de cercles "usuel" mais tel que la réunion des disques fermés bordés par les cercles ne couvre pas la sphère. Les motifs de cercles hyperidéaux sont directement liés aux polyèdres hyperboliques hyperidéaux, et aussi aux empilements de cercles.

A chaque motif de cercles hyperidéal, on associe un graphe d'incidence et un ensemble d'angles d'intersection. On caractérise les graphes d'incidence et les angles d'intersection possibles dans la sphère, le tore, et sur les surfaces de genre supérieur. C'est une conséquence d'un résultat plus général décrivant les motifs de cercles hyperidéaux dans les bords de variétés hyperboliques géométriquement finies (pour les $\mathbb{C} P^{1}$-structures correspondantes). Ce résultat plus général est obtenu comme conséquence d'un théorème d'Otal [Ota94, BO01] sur les laminations de plissage des coeurs convexes de variétés hyperboliques géométriquement finies.

\section{Introduction}

1.1. Circle patterns. A circle packing on the sphere $S^{2}$ is a finite family of oriented circles with disjoint interiors. The incidence graph of a circle packing is a graph $\Gamma$, embedded in $S^{2}$, which has one vertex for each circle, and an edge between two vertices when the corresponding circles are tangent. A classical theorem of Koebe [Koe36] states that, given any graph $\Gamma$ on $S^{2}$ which is the 1-skeleton of a polytopal triangulation, there is a unique circle packing with incidence graph $\Gamma$ - the uniqueness is up to Möbius transformations. This theorem was extended to higher genus surfaces by Thurston [Thu80]. It also

Received July 27, 2004. Revised October 2004. 
holds when $\Gamma$ is the 1-skeleton of a cellular decomposition; one should then add the condition that, for each connected component of the complement of the disks, there is another circle orthogonal to all the adjacent circles of the pattern.

One can also consider circle patterns on $S^{2}$ (see e.g. [BS04]). For reasons that should become apparent below, we add the adjective ideal to describe what is perhaps the most commonly considered type of circle patterns.

Definition 1.1. A circle pattern on $S^{2}$ is a finite family of oriented circles $C_{1}, \cdots, C_{N}$. Given a circle pattern, an interstice is a connected component of the complement of the union of the open disks bounded by the circles. If $C_{1}, \cdots, C_{N}$ is a circle pattern, it is ideal if:

- Each interstice is a point.

- If $D$ is an open disk in $S^{2}$, containing no interstice, but such that its closure contains at least 3 of the interstices, then $D$ is the open disk bounded by one of the $C_{i}, 1 \leq i \leq N$.

It follows from this definition that any interstice is in at least 3 of the circles. However there might also be points which are in 3 circles or more, but which are not interstices.

Given an ideal circle pattern, its incidence graph is the 1-skeleton of the cellular decomposition of the sphere which has:

- One vertex for each circle.

- One face for each interstice.

- One edge between two vertices, when the corresponding circles intersect at two interstices.

Given two of the circles in an ideal circle pattern, we consider the angle between them only when the corresponding vertices of the incidence graph share an edge. Thus the intersection angles between the circles correspond to a function from the set of edges of the incidence graph to $(0, \pi)$. The angle will always be measured in the complement of one disk in the other, i.e. it will be equal to $\pi$ minus the intersection angle measured in the intersection of the two disks. By extension, the intersection angle between the boundaries of two disjoint but tangent open disks is equal to $\pi$.

The notion of ideal circle pattern is not restricted to the sphere; one can consider it in an Euclidean or a hyperbolic surface. Actually the most natural setting is a surface with a $\mathbb{C} P^{1}$-structure, since the notion of circle is then well defined, as well as the notion of angle. A surface with a spherical, Euclidean or hyperbolic metric also has a canonical $\mathbb{C} P^{1}$-structure coming from the Poincaré uniformization theorem.

1.2. Ideal polyhedra. An ideal hyperbolic polyhedron is a convex polyhedron in $H^{3}$, of finite volume, with all its vertices on the sphere at infinity. Another possible definition is as the convex hull of a finite set of points (not all contained in a plane) on the sphere at infinity in $H^{3}$.

The possible combinatorics and dihedral angles of ideal polyhedra were described by Andreev [And71] and Rivin [Riv96]. Thurston [Thu80] realized that 


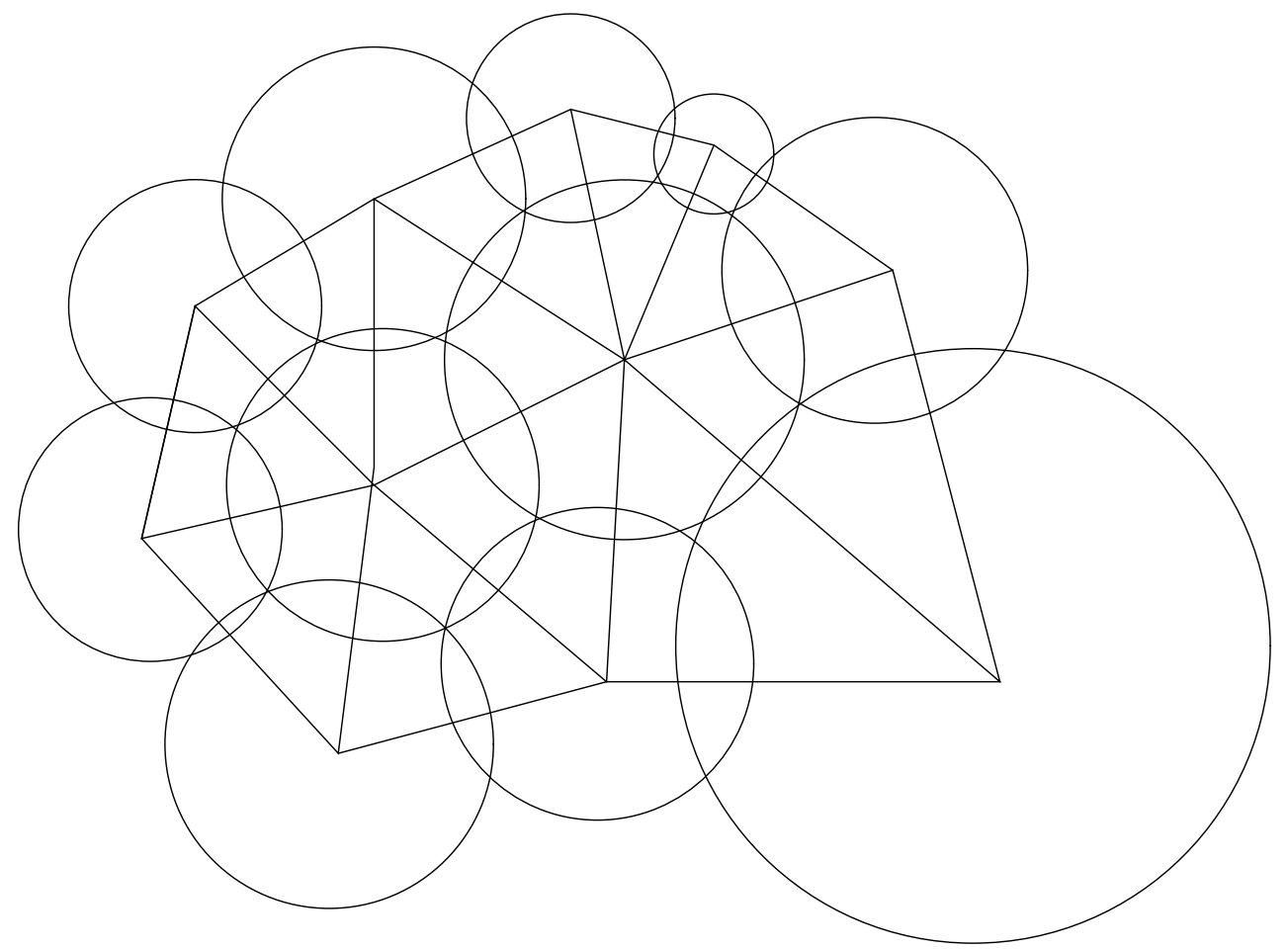

Figure 1. A piece of ideal circle pattern, with its incidence graph.

there was a deep connection between ideal polyhedra and circle patterns: given an ideal polyhedron, the boundaries of the hyperbolic planes containing its faces is an ideal circle pattern; its incidence graph is combinatorially dual to the combinatorics of the ideal polyhedron, and the exterior dihedral angles between the faces are equal to the intersection angles between the circles. Using this, Thurston gave a simple proof of the Koebe circle packing theorem based on Andreev's theorem. Thus Rivin's theorem on ideal polyhedra can be used to describe the possible combinatorics and dihedral angles of ideal circle patterns, in a way which is similar to Theorem 1.3 below. Many interesting extensions and important references can be found in [BS04, Riv03, Lei02], in particular regarding the extension to higher genus surfaces.

1.3. Hyperideal circle patterns. We now introduce another notion of circle patterns. Just as the ideal circle patterns are related to ideal hyperbolic polyhedra, those other circle patterns are related to strictly hyperideal polyhedra, so we call them strictly hyperideal circle patterns.

Definition 1.2. Let $C_{1}, \cdots, C_{N}$ be a circle pattern in $S^{2}$, with interstices $I_{1}, \cdots, I_{M}$. It is strictly hyperideal if:

- Each interstice has non-empty interior.

- For each $j \in\{1, \cdots, M\}$, there is an oriented circle $C_{j}^{\prime}$, containing $I_{j}$, which is orthogonal to all the circles $C_{i}$ adjacent to $I_{j}$. 
- For all $i \in\{1, \cdots, N\}$ and all $j \in\{1, \cdots, M\}$, if $C_{i}$ is not adjacent to $I_{j}$, then either the interior of $C_{i}$ is disjoint from the interior of $C_{j}^{\prime}$, or $C_{i}$ intersects $C_{j}^{\prime}$ and their intersection angle is strictly larger than $\pi / 2$.

- If $D$ is an open disk in $S^{2}$ such that:

(1) For each $j \in\{1, \cdots, M\}$, either $D$ is disjoint from the interior of $C_{j}^{\prime}$, or $\partial D$ has an intersection angle at least $\pi / 2$ with $C_{j}^{\prime}$.

(2) $\partial D$ is orthogonal to at least 3 of the $C_{j}^{\prime}$. then $\partial D$ is one of the $C_{i}$.

As for ideal circle patterns, this notion is not restricted to the sphere, but can be considered for any surface with a $\mathbb{C} P^{1}$-structure. In this setting, it should be understood that, in the last condition, $D$ is a disk which is immersed in the surface, i.e. not necessarily embedded.

Taking the limit of such strictly hyperideal circle patterns as the radii of the $C_{j}^{\prime}$ goes to zero yields ideal circle patterns. On the other hand, taking the limit as the intersection angles between the $C_{i}$ goes to $\pi$ yields a circle packing as in the Koebe theorem quoted above. There is a natural notion which generalizes both the ideal and the strictly hyperideal circle patterns, and the name "hyperideal circle pattern" should be kept for this more general notion.

Given a strictly hyperideal circle pattern, its incidence graph is the 1-skeleton of the cellular decomposition of $S^{2}$ defined similarly as for ideal circle patterns, which has:

- One face for each of the $I_{j}, 1 \leq j \leq M$.

- One vertex for each of the $C_{i}, 1 \leq i \leq N$.

- One edge between two faces, corresponding to $I_{k}$ and $I_{l}$, whenever there are two circles $C_{i}$ and $C_{j}$ which are both orthogonal to $C_{k}^{\prime}$ and $C_{l}^{\prime}$.

We will consider the intersection angles between the circles $C_{i}$, defined as explained above for ideal circle patterns.

1.4. Some examples. Before stating the main result, we give three simpler examples, two of them corresponding to already well understood cases. The first is a direct consequence of a recent result of Bao and Bonahon [BB02].

Theorem 1.3. Let $\Gamma$ be the 1-skeleton of a polytopal cellular decomposition of $S^{2}$, and let $w: \Gamma_{1} \rightarrow(0, \pi)$ be a map on the set of edges of $\Gamma$. There exists a strictly hyperideal circle pattern on $S^{2}$ with incidence graph $\Gamma$ and intersection angles given by $w$ if and only if:

(1) For each simple closed curve $\gamma$ in $\Gamma$, the sum of the values of $w$ on the edges of $\gamma$ is strictly larger than $2 \pi$.

(2) For each open path $\gamma$ in $\Gamma$, which begins and ends on the boundary of a face $f$ but is not contained in $f$, the sum of the values of $w$ on the edges of $\gamma$ is strictly larger than $\pi$.

This strictly hyperideal circle pattern is then unique, up to the Möbius transformations of $S^{2}$.

There is a similar result in the case of the torus. 


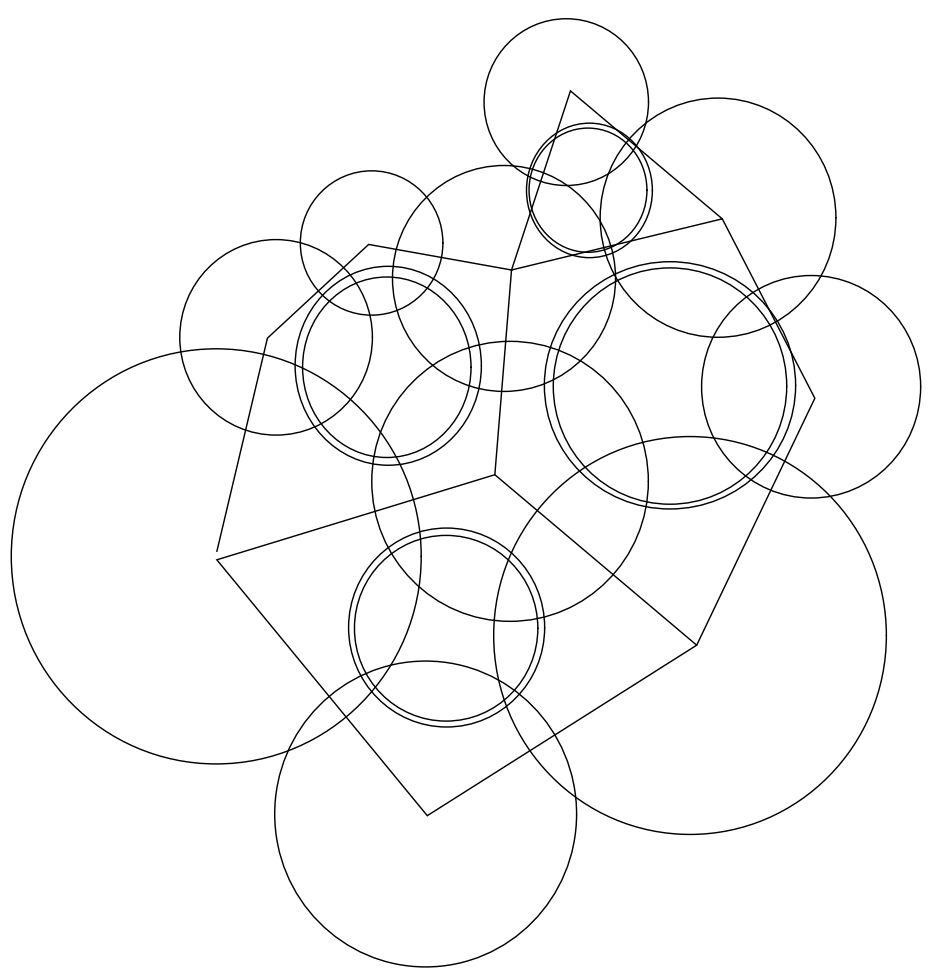

Figure 2. A piece of hyperideal circle pattern, with its incidence graph. The circles corresponding to the interstices are doubled. The angles between the circles of the two families should be $\pi / 2$.

Theorem 1.4. Let $\Gamma$ be the 1-skeleton of a cellular decomposition of $T^{2}$, and let $w: \Gamma_{1} \rightarrow(0, \pi)$ be a map on the set of edges of $\Gamma$. There exists a flat metric $g_{0}$ on $T^{2}$ and a strictly hyperideal circle pattern $C$ on $\left(T^{2}, g_{0}\right)$ with incidence graph $\Gamma$ and intersection angles given by $w$ if and only if:

(1) For each simple, homotopically trivial closed path $\gamma$ in $\Gamma$, the sum of the values of $w$ on the edges of $\gamma$ is strictly larger than $2 \pi$.

(2) For each open path $\gamma$ in $\Gamma$, which begins and ends on the boundary of a face $f$, is homotopic to a segment in that face, but is not contained in $f$, the sum of the values of $w$ on the edges of $\gamma$ is strictly larger than $\pi$.

Then $\left(g_{0}, C\right)$ is unique, up to the homotheties of $g_{0}$.

The same statement holds on surfaces of higher genus, it is a direct consequence of (a special case of) a recent result of Rousset [Rou04].

Theorem 1.5. Let $S_{g}$ be a closed surface of genus $g \geq 2$, let $\Gamma$ be the 1-skeleton of a cellular decomposition of $S_{g}$, and let $w: \Gamma_{1} \rightarrow(0, \pi)$ be a map on the set of edges of $\Gamma$. There exists a hyperbolic metric $g_{0}$ on $S_{g}$ and a strictly hyperideal circle pattern $C$ on $\left(S_{g}, g_{0}\right)$ with incidence graph $\Gamma$ and intersection angles given by $w$ if and only if: 
(1) For each simple, homotopically trivial closed path $\gamma$ in $\Gamma$, the sum of the values of $w$ on the edges of $\gamma$ is strictly larger than $2 \pi$.

(2) For each open path $\gamma$ in $\Gamma$, which begins and ends on the boundary of a face $f$, is homotopic to a segment $f$, but is not contained in $f$, the sum of the values of $w$ on the edges of $\gamma$ is strictly larger than $\pi$.

Then $\left(g_{0}, C\right)$ is unique.

1.5. The main result. The 3 theorems quoted above are basically simple consequences of the following more complex but much more general statement. We now consider a compact 3-manifold with non-empty boundary $M$, whose interior admits a complete hyperbolic metric; $M$, like all the manifolds that we consider in this paper, will be oriented. According to a result of Thurston [Thu82], the existence of a complete hyperbolic metric on the interior of $M$ is equivalent to a simple topological condition: that $M$ is irreducible and homotopically atoroidal, and is not the interval bundle over the Klein bottle.

We call $\partial^{\prime} M$ the union of the connected components of $\partial M$ which are not tori, except if $M$ is a solid torus - then $\partial^{\prime} M=\partial M$ - or if $M$ is the product of a torus by an interval - then $\partial^{\prime} M$ is one connected component of $\partial M$.

In this setting, the complete hyperbolic metrics on $M$ are geometrically finite; they contain a finite volume subset $C$ which is convex in the (strong) sense that, given any geodesic segment $\gamma$ with endpoints in $C, \gamma$ is contained in $C$ (see [Thu80]). For each such metric, $M$ is isometric to the quotient of $H^{3}$ by a discrete group $G$ acting by isometries. Then $\partial^{\prime} M$ is the quotient of the discontinuity domain of $G$ by $G$. Since $G$ acts on $S^{2}$ by Möbius transformations, $\partial^{\prime} M$ has a natural $\mathbb{C} P^{1}$-structures. Note that, in general, among the $\mathbb{C} P^{1}$-structures on $\partial^{\prime} M$, only a small subset are obtained from a geometrically finite hyperbolic metric on $M$.

Finally, before stating the result, we need a small restriction on the kind of cellular decomposition which can be realized.

Definition 1.6. Let $M$ be a compact 3-manifold with boundary, whose interior admits a complete hyperbolic metric. Let $\Sigma$ be a cellular decomposition of $\partial^{\prime} M$. $\Sigma$ is proper if there is no essential disk $D$ in $M$ such that $\partial D \subset \partial^{\prime} M$ and that $\partial D$ intersects the closure of at most two cells.

Theorem 1.7. Let $M$ be a compact (orientable) 3-manifold with boundary whose interior admits a complete hyperbolic metric. Let $\Gamma$ be the 1-skeleton of a proper cellular decomposition $\Sigma$ of $\partial^{\prime} M$, and let $w: \Gamma_{1} \rightarrow(0, \pi)$ be a map on the set of edges of $\Gamma$. There exists a couple $(\sigma, C)$, where $\sigma$ is a $\mathbb{C} P^{1}$-structure on $\partial^{\prime} M$ induced by a geometrically finite hyperbolic metric on $M$ and $C$ is a strictly hyperideal circle pattern on $\left(\partial^{\prime} M, \sigma\right)$ with incidence graph $\Gamma$ and intersection angles given by $w$, if and only if:

(1) The cellular decomposition $\Sigma$ is proper.

(2) For each simple closed path $\gamma$ in $\Gamma$, homotopically trivial in $M$, the sum of the values of $w$ on the edges of $\gamma$ is strictly larger than $2 \pi$. 
(3) For each open path $\gamma$ in $\Gamma$, which begins and ends on the boundary of a face $f$, is homotopic in $M$ to a segment in $f$, but is not contained in $f$, the sum of the values of $w$ on the edges of $\gamma$ is strictly larger than $\pi$.

Then $(\sigma, C)$ is unique.

Theorems 1.3, 1.4 and 1.5 are direct consequences of Theorem 1.7. Theorem 1.3 is obtained when $M$ is a ball, and Theorem 1.4 when $M$ is the product of a torus by $\mathbb{R}$. For Theorem 1.5, one should take as $M$ the product of a surface of genus at least 2 by an interval, with a graph $\Gamma$ and a function $w$ which are the same on both connected components of the boundary; the uniqueness in Theorem 1.7 then shows that the $\mathbb{C} P^{1}$-structure obtained on each connected component of the boundary is the same, so that it is the $\mathbb{C} P^{1}$-structure induced by a hyperbolic metric on the surface considered.

There are other simple consequences of Theorem 1.7 beyond the three statements given above. For instance, one can consider two cellular decompositions of a surface $\Sigma$ of genus at least 2, along with some angles on the edges, and obtain from Theorem 1.7 a unique quasi-fuchsian metric on $\Sigma \times \mathbb{R}$, along with one strictly hyperideal circle pattern on each boundary component.

1.6. Relation to other results. Theorem 1.7 is similar to the main result of [Sch02], although [Sch02] is about hyperideal polyhedra rather than hyperideal circle patterns. Theorem is 1.7 less general than the main result of [Sch02] insofar as the circle patterns considered are strictly hyperideal rather than hyperideal (a more general notion), but also more general since $M$ can have compressible boundary and $\partial M$ is allowed to have toric components. The proof of Theorem 1.7, however, is completely different from the one given in [Sch02]; here we show that Theorem 1.7 is a direct consequence of a result of Otal [Ota94, BO01], which itself uses crucially a result of Hodgson and Kerckhoff [HK98]. In [Sch02], the proof is direct and based, among other things, on some properties of the volume of hyperideal polyhedra. The method of [Sch02] yields additional informations, in particular concerning the "induced metrics" on the boundaries of the manifolds with hyperideal boundary that appear here in section 2 .

\section{Circle patterns and hyperideal polyhedra}

In this section we define "manifolds with strictly hyperideal boundary" and state a theorem, concerning them, which is basically equivalent to Theorem 1.7. This theorem is proved in the next section, using [Ota94, BO01].

2.1. Hyperideal polyhedra. Recall that the Klein model of $H^{3}$ is a map $\phi: H^{3} \rightarrow B^{3}$, where $B^{3}$ is the open unit ball in $\mathbb{R}^{3}$, which is projective, i.e. it sends the hyperbolic geodesics to the segments. A hyperideal polyhedron is a hyperbolic polyhedron which is the inverse image, in $H^{3}$, of a polyhedron $P \subset \mathbb{R}^{3}$ with all its vertices outside $B^{3}$ but all its edges intersecting $B^{3}$. It is strictly hyperideal if $P$ has no vertex on the boundary of $B^{3}$. 
Given a point $v \in \mathbb{R}^{3} \backslash \overline{B^{3}}$, its polar dual is the plane, noted $v^{*}$, which contains the set of points $x \in S^{2}$ such that the line going through $x$ and $v$ is tangent to $S^{2}$. (It can also be defined using the polarity with respect to a bilinear form of signature $(3,1)$ on $\mathbb{R}^{4}$, which explains the terminology.) An important property is that, for any $x \in v^{*} \cap B^{3}$, the intersection with $B^{3}$ of the line going through $x$ and $v$, considered as a hyperbolic geodesic, is orthogonal to $v^{*} \cap B^{3}$, considered as a hyperbolic plane. Conversely, for any plane $P \subset \mathbb{R}^{3}$ intersecting $B^{3}$, there is a unique point $P^{*} \in \mathbb{R}^{3} \backslash B^{3}$ such that $P$ is the plane dual to $v$. This notion of duality has many interesting application, it is related to the hyperbolic-de Sitter duality used in particular by Rivin [Riv86] and Rivin and Hodgson [RH93] to obtain beautiful results on compact hyperbolic polyhedra. To simplify statements a little, we will sometimes talk about the point which is dual to an oriented circle in $S^{2}$; it is the point dual to the plane which contains the circle.

Using this notion, we can reformulate the definition of a hyperideal polyhedron without reference to the projective model of $H^{3}$; a hyperideal polyhedron is a convex hyperbolic polyhedron (i.e. the intersection of a finite number of halfplanes) with no vertex such that, for each end, either the end has finite volume, or there exists a hyperbolic plane which is orthogonal to all the edges going to infinity in it. It is strictly hyperideal if all ends have infinite volume.

Moreover, given a hyperideal polyhedron $P$, one can truncate it (following [BB02]) by cutting off each end of infinite volume by the plane which is dual to the corresponding vertex; one obtains in this way a finite volume polyhedron $P_{t}$ (which is compact if $P$ is strictly hyperideal). Its faces are either faces of $P$, or faces which are orthogonal to the adjacent faces.

2.2. Ideal polyhedra and ideal circle patterns. We first recall the correspondence between ideal hyperbolic polyhedra and ideal circle patterns. It is a direct consequence of the following proposition, which is classical, see e.g. [GW93].

Proposition 2.1. Let $x_{1}, \cdots, x_{M} \in S^{2}$ be distinct points, $M \geq 3$. There exists a unique family of oriented circles $C_{1}, \cdots, C_{N}$ such that:

- The $C_{i}$ bound closed disks which cover $S^{2}$.

- None of the $C_{i}$ bounds an open disk containing any of the $x_{j}$.

- Each of the $C_{i}$ contains at least 3 of the $x_{j}$.

- If $D$ is an open disk which contains none of the $x_{j}$ but such that $\partial D$ contains at least 3 of them, then $D$ is the interior of one of the $C_{i}$.

The $C_{j}$ are obtained as the intersections with $S^{2}$ of the planes containing the faces of the convex hull, in $\mathbb{R}^{3}$, of the points $x_{1}, \cdots, x_{M}$.

This proposition explains the relationship between ideal polyhedra and ideal circle patterns. Given a (convex) ideal polyhedron, it is clear that the set of oriented circles associated to its faces is an ideal circle pattern. Conversely, given an ideal circle pattern $C_{1}, \cdots, C_{N}$, let $x_{1}, \cdots, x_{M}$ be its interstices. Then Definition 1.1 implies that $C_{1}, \cdots, C_{N}$ is the set of oriented circles associated 
by Proposition 2.1 to $x_{1}, \cdots, x_{M}$. Therefore $C_{1}, \cdots, C_{N}$ are the boundaries of the planes containing the faces of the ideal polyhedron which is the convex hull of the $x_{j}$.

2.3. Hyperideal polyhedra and hyperideal circle patterns. The same procedure can be applied for strictly hyperideal circle patterns, based on the following analog of Proposition 2.1 (see [GW93]).

Proposition 2.2. Let $C_{1}^{\prime}, \cdots, C_{M}^{\prime}$ be a family of oriented circles in $S^{2}$, bounding disjoint closed disks ( $M \geq 3$ ), each disk being strictly smaller than a hemisphere. There exists a unique family of oriented circles $C_{1}, \cdots, C_{N}$ such that:

- The closed disks bounded by the $C_{i}$ cover the complement of the closed disks bounded by the $C_{j}^{\prime}$.

- None of the $C_{i}$ contains any of the $C_{j}^{\prime}$ in its interior, or makes an angle strictly less than $\pi / 2$ with any of the $C_{j}^{\prime}$.

- Each of the $C_{i}$ is orthogonal to at least 3 of the $C_{j}^{\prime}$.

- If $D$ is an open disk such that:

(1) For each $j \in\{1, \cdots, M\}$, either $D$ is disjoint from the interior of $C_{j}^{\prime}$, or $\partial D$ makes an angle at least $\pi / 2$ with $C_{j}^{\prime}$.

(2) $\partial D$ is orthogonal to at least 3 of the $C_{j}^{\prime}$.

then $D$ is the interior of one of the $C_{i}$.

The $C_{i}$ are obtained as the intersections with $S^{2}$ of the planes containing the faces of the convex hull, in $\mathbb{R}^{3}$, of the points $x_{1}, \cdots, x_{M}$ which are dual to the circles $C_{1}^{\prime}, \cdots, C_{M}^{\prime}$.

Clearly the hypothesis that the circles $C_{j}^{\prime}$ bound disks smaller than hemispheres is not crucial, it appears here in the convex hull construction because we do things in the Euclidean space rather than in the sphere, where statements would be a little more complicated. Note also that there is a common generalization of Proposition 2.1 and Proposition 2.2, see [GW93].

Given a strictly hyperideal polyhedron $P$, one can consider the oriented planes containing its faces, and then their boundaries, which are oriented circles in $S^{2}$. Those circles clearly make up a strictly hyperideal circle pattern, with the connected components of the complement of the disks corresponding to the vertices of $P$; the circles $C_{j}^{\prime}$ which appear in the definition of a strictly hyperideal circle pattern are the boundaries of the hyperbolic planes dual to the strictly hyperideal vertices. Moreover, the incidence graph of this circle pattern is dual to the combinatorics as the polyhedron $P$, because two faces of the incidence graph - corresponding to two connected components of the complement of the disks - are adjacent if and only if the corresponding circles are both orthogonal to two circles of the pattern, i.e. if and only if the corresponding hyperideal vertices of $P$ are adjacent.

Conversely, let $C=\left(C_{1}, \cdots, C_{N}\right)$ be a strictly hyperideal circle pattern. Let $C_{1}^{\prime}, \cdots, C_{M}^{\prime}$ be the oriented circles corresponding to the interstices. Since those circles bound disjoint disks, we can apply a Möbius transformation to ensure that all those disks are strictly smaller than hemispheres. 
Definition 1.2 shows that the circles $C_{1}, \cdots, C_{N}$ are precisely the circles associated to $C_{1}^{\prime}, \cdots, C_{M}^{\prime}$ by Proposition 2.2. Therefore, the $C_{i}$ are the boundaries of the planes containing the faces of a strictly hyperideal polyhedron, which is the convex hull of the points dual to the $C_{j}^{\prime}$.

2.4. Manifolds with hyperideal boundary. It is necessary below to consider objects, more general than hyperideal polyhedra, which are basically submanifolds $M$ of a geometrically finite hyperbolic 3-manifold $N$ such that the boundary of $M$ in $N$ is locally like a strictly hyperideal polyhedron in $H^{3}$.

A geometrically finite hyperbolic 3-manifold is the interior of a compact 3manifold with boundary $N$ with a complete hyperbolic metric, which contains a non-empty submanifold $M \subset N$, of finite volume, which is convex in the (strong) sense that any geodesic segment in $N$ with endpoints in $M$ actually remains in $M$. Moreover, if $N$ is not a ball or the product of a torus by an interval, there exists a smallest such subset $M_{0}$ (smallest with respect to the inclusion) and $M_{0}$ is called the convex core of $N$. If $N$ is not a ball, a solid torus or the product of the torus by an interval, then the boundary of $M_{0}$ in $N$ is homeomorphic to the union of the connected components of $\partial N$ which are not tori, while the tori in the boundary of $N$ correspond to cusps in $M_{0}$ (see e.g. [Thu82, Thu80] for much more on this).

Definition 2.3. A hyperbolic manifold with strictly hyperideal boundary is the interior of a compact 3-manifold with boundary $M$ with a hyperbolic metric, such that there exists an isometric embedding $\phi: M \rightarrow N$, where $N$ is a geometrically finite hyperbolic 3-manifold, with the property that:

- $\phi(M)$ is convex in $N$ in the strong sense defined above: any geodesic segment of $N$ with endpoints in $\phi(M)$ actually remains in $\phi(M)$.

- For any embedded hyperbolic ball $B \subset N$, there exists a strictly hyperideal polyhedron $P \subset H^{3}$ and a ball $B^{\prime} \subset H^{3}$ such that the interior of $B^{\prime} \cap P$ is isometric to $B \cap \phi(M)$.

- The boundary of $\phi(M)$ in $N$ contains no closed geodesic of $N$.

Given $M, N$ is uniquely defined and will be called the extension of $M$.

In many cases it is more convenient to consider the closure of $M$ in $N$, rather than $M$ as defined above. The condition on the closed geodesics is equivalent to the fact that the boundary of $M$ in $N$ does not intersect the convex core of $N$. There is another possible definition, using the notion of hyperideal point in a geometrically finite manifold. A simple example of a strictly hyperideal manifold is that, when $N$ is the ball, $M$ is simply a strictly hyperideal polyhedron.

2.5. Dihedral angles of manifolds with hyperideal boundary. Consider a hyperbolic manifold $M$ with strictly hyperideal boundary. By definition, its boundary $\partial M$ is a "polyhedral" surface in the extension $N$ of $M$. It has a finite number of faces and a finite number of edges. Each face is isometric to the interior of a strictly hyperideal polygon in $H^{2}$, while the edges are complete geodesics. The boundary combinatorics of $M$ determines (combinatorially) a 
cellular decomposition of $\partial^{\prime} N$ - with one face for each face of $\partial M$, and one vertex for each strictly hyperideal vertex of $M$ (or, equivalently, for each end of $\partial M)$.

For each edge $e$ of $\partial M$, we define the exterior dihedral angle of $\partial M$ at $e$ to be $\pi$ minus the angle, measured in $M$, between the two faces containing $e$. Another possible definition is as the angle, measured on $e$, between the exterior normal vectors to the two faces of $\partial M$ containing $e$. The possible combinatorics and boundary angles of the strictly hyperideal manifolds which have as their extension a given geometrically finite hyperbolic 3-manifold $N$ are described in the following statement. It has non-empty intersection with the results of [BB02, Rou04, Sch02].

Theorem 2.4. Let $N$ be compact 3-manifold with boundary, whose interior admits a complete hyperbolic metric. Let $\Gamma$ be a graph, embedded in $\partial^{\prime} N$, which is the 1-skeleton of a cellular decomposition $\Sigma$ of $\partial^{\prime} N$, and let $w: \Gamma_{1} \rightarrow(0, \pi)$ be a map from the set of edges of $\Gamma$ to $(0, \pi)$. There exists a strictly hyperideal hyperbolic manifold $M$, which can be isometrically embedded in $N$ with some geometrically finite metric, with boundary combinatorics given by $\Gamma$ and exterior dihedral angles described by $w$, if and only if:

(1) The cellular decomposition $\Sigma$ is proper.

(2) For each simple closed path $\gamma$ in the dual graph $\Gamma^{*}$ of $\Gamma$, which is homotopically trivial in $M$, the sum of the values of $w$ on the edges of $\gamma$ is strictly larger than $2 \pi$.

(3) For each open path $c$ in $\Gamma^{*}$ which begins and ends on the same face $f$ of $\Gamma^{*}$, is not contained in the boundary of $f$, but is homotopic in $M$ to a segment in $f$, the sum of the values of $w$ on the edges of $c$ is strictly larger than $\pi$.

This manifold $M$ is then unique.

Note that the existence of a function $w$ satisfying the requested conditions implies constraints on $\Gamma$; for instance, if $M$ is a ball, $\Gamma$ has to be the 1-skeleton of a polytopal cellular decomposition (see [BS04, Sch02] for more details on this).

2.6. Proof of Theorem 1.7 from Theorem 2.4. Let $N, \Gamma$ and $w$ be given as in the statement of Theorem 1.7. Apply Theorem 2.4 to obtain a strictly hyperideal hyperbolic metric $h$ on $N$ and a strictly hyperideal manifold $M$ isometrically embedded in $(N, h)$. Let $\sigma$ be the $\mathbb{C} P^{1}$-structure induced on $\partial^{\prime} N$ by $(N, h)$, and let $C$ be the strictly hyperideal circle pattern defined on $\partial^{\prime} N$ by the boundaries of the planes containing the faces of $M$. By construction, $C$ has the combinatorics dual to the combinatorics of $\Gamma$ and the intersection angles determined by $w$. Moreover, the uniqueness in Theorem 1.7 will follow from the uniqueness in Theorem 2.4 if we show that any strictly hyperideal circle pattern in $\partial^{\prime} N$, for a $\mathbb{C} P^{1}$-structures which is induced by a geometrically finite metric on $N$, comes from a strictly hyperideal hyperbolic manifold isometrically embedded in $N$ (with some complete hyperbolic metric, not necessarily $h$ ). 
Let $\sigma$ be a $\mathbb{C} P^{1}$-structure on $\partial^{\prime} N$ induced by a geometrically finite hyperbolic metric $g$ on $N$, and let $C$ be a strictly hyperideal circle pattern in $\partial^{\prime} N$ for $\sigma$. Let $\tilde{g}$ be the lift of $g$ to the universal cover $\tilde{N}$ of $N$; then $(\tilde{N}, \tilde{g})$ is isometrically identified with $H^{3}$, and the fundamental group $\pi_{1} N$ of $N$ acts on $H^{3}$ by isometries. Let $\Lambda \subset S^{2}$ be its limit set.

Under the projection from $\tilde{N}$ to $N, \partial^{\prime} N$ lifts to the complement of $\Lambda$ in $S^{2}$. The strictly hyperideal circle pattern $C$ lifts to an infinite set $\tilde{C}$ of circles in $S^{2} \backslash \Lambda$, which accumulates close to $\Lambda$ - each neighborhood of a point of $\Lambda$ contains an infinite number of circles. However $\tilde{C}$ is still a strictly hyperideal circle pattern in the sense of Definition 1.2 (except of course for the finiteness condition). The other set of oriented circles appearing in the definition of a hyperideal circle pattern, say $C^{\prime}=\left\{C_{1}^{\prime}, \cdots, C_{M}^{\prime}\right\}$, lifts to a set $\tilde{C}^{\prime}$ of disjoint circles in $S^{2} \backslash \Lambda$, which also accumulates close to $\Lambda$.

Now consider the images of $\tilde{C}$ and $\tilde{C}^{\prime}$ by the projective map $\phi$ of the Klein model, which we call $\tilde{C}_{e}$ and $\tilde{C}_{e}^{\prime}$, respectively. We suppose that each circle of $\tilde{C}_{e}^{\prime}$ bounds a disk smaller than a hemisphere (this can be achieved by applying a projective transformation since the circles of $\tilde{C}_{e}^{\prime}$ bound disjoint disks). For each $c^{\prime} \in \tilde{C}_{e}^{\prime}$, let $s\left(c^{\prime}\right)$ be the point in $\mathbb{R}^{3}$ which is dual to the oriented plane $p^{\prime}$ such that $p^{\prime} \cap S^{2}=c^{\prime}$. For each $c \in \tilde{C}_{e}$, let $h(c)$ be the closed half-space, bounded by the plane containing $c$, which contains $\phi(\Lambda)$. The elementary properties of the duality indicate that, for all $c \in \tilde{C}_{e}$ and all $c^{\prime} \in \tilde{C}_{e}^{\prime}$ :

- $s\left(c^{\prime}\right) \in h(c)$ if and only if either the interior of $c^{\prime}$ is disjoint from the interior of $c$, or $c$ and $c^{\prime}$ intersect with angle at least $\pi / 2$.

- $s\left(c^{\prime}\right) \in \partial h(c)$ if and only if $c$ and $c^{\prime}$ are orthogonal.

Remark 2.5. The intersection of the $h(c)$, for $c \in \tilde{C}_{e}$, is equal to the convex hull of the $s\left(c^{\prime}\right)$, for $c^{\prime} \in \tilde{C}_{e}^{\prime}$.

Proof. Let $U:=\cap_{c \in \tilde{C}_{e}} h(c)$. By definition, $U$ is a convex subset of $\mathbb{R}^{3}$. Moreover, for each $c^{\prime} \in \tilde{C}_{e}^{\prime}, c^{\prime}$ is orthogonal to at least 3 of the circles in $\tilde{C}_{e}$, so that $s\left(c^{\prime}\right)$ is in $\partial h(c)$ for at least 3 elements of $\tilde{C}_{e}$. Since $s\left(c^{\prime}\right)$ is contained in $h(c)$ for all $c \in \tilde{C}_{e}$, it follows that $s\left(c^{\prime}\right)$ is an extreme point of $U$.

Conversely, let $s_{0}$ be an extreme point of $U$. If $s_{0} \in \phi(\Lambda)$, then $s_{0}=$ $\lim _{n \rightarrow \infty} s\left(c_{n}^{\prime}\right)$, for a sequence $c_{n}^{\prime}$ in $\tilde{C}_{e}^{\prime}$. Otherwise, $s_{0}$ has to be outside the closed ball of radius 1 . Let $p_{0}$ be the dual oriented plane, let $c_{0}=p_{0} \cap S^{2}$, and let $D_{0}$ be the open disk bounded by $c_{0}$. Then, for all $c \in \tilde{C}_{e}, s_{0}$ is not in the interior of $h(c)$, so that either $D_{0}$ is disjoint from the interior of $c$, or $\partial D_{0}$ has an intersection angle at least $\pi / 2$ with $c$. In addition, since $s_{0}$ is an extreme point of $U$, there exist at least 3 elements $c \in \tilde{C}_{e}$ such that $s_{0} \in \partial h(c)$, so there exists at least 3 elements $c \in \tilde{C}_{e}$ such that $\partial D_{0}$ is orthogonal to $c$. Going back to $\tilde{N}$ and then projecting to $N$, this contradicts the last point of the definition of a hyperideal circle packing, Definition 1.2.

Let $S:=\left\{s\left(c^{\prime}\right) \mid c^{\prime} \in \tilde{C}_{e}^{\prime}\right\}$. By definition, $S$ is invariant under the action of $\pi_{1} N$ on $\mathbb{R}^{3}$ (which is by projective transformations preserving $S^{2}$ ), so that its 
convex hull is also invariant under the action of $\pi_{1} N$. Taking the quotient of the convex hull of $S$ by the action of $\pi_{1} N$ defines, in $N$, a manifold with strictly hyperideal boundary, whose combinatorics and boundary angles are as needed. Thus Theorem 1.7 follows from Theorem 2.4.

\section{Hyperideal polyhedra and hyperbolic convex cores}

It remains, in this section, to prove Theorem 2.4. We will show here that it is a simple consequence of a result of Otal [Ota94, BO01].

3.1. Convex cores of hyperbolic manifolds. Let $N$ be a geometrically finite hyperbolic 3-manifold (not a ball, a solid torus or the product of a torus by an interval). We have defined above the convex core of $N$ as the smallest nonempty subset of $N$ which is convex in the sense that, given a geodesic segment $\gamma$ in $N$ with endpoints in $C, \gamma \subset C$. The convex core of $N$ has finite volume (by definition of a geometrically finite hyperbolic manifold).

The boundary of the convex core of $N$ is a non-smooth surface in $N$ which is locally convex; it has a hyperbolic induced metric, but is "bent" along a lamination (see [Thu80]), called the "pleating lamination". The precise way the bending occurs is described by a transverse measure defined on the pleating lamination. We are interested here only in a very special case, where the support of the pleating lamination is a disjoint union of simple closed curves; in this case we say that the pleating lamination is rational.

In this case, the boundary of the convex core is a polyhedral-like surface. It has totally geodesic faces, edges which are closed geodesics (corresponding to the curves in the support of the pleating lamination) and no vertex. To each edge is associated a number — describing the weight of the corresponding leaf for the transverse measure of the pleating lamination — which is simply the angle between the oriented normals of the "faces" on the two sides; see e.g. [EM86, Bon01] for more on the local geometry of the boundary of the convex core.

It follows from its definition that the convex core of $N$ has the same topology as $N$. Since the pleating lamination and its bending measure are topological in nature, we can consider them on the boundary of $N$ rather than the boundary of its convex core.

3.2. A theorem of Otal. The possible transverse measures of the rational pleating laminations of hyperbolic convex cores are precisely described by the following result.

Theorem 3.1 (Otal [Ota94, BO01]). Let $N$ be a compact 3-manifold with boundary, whose interior admits a complete hyperbolic metric. Let $\alpha$ be a measured lamination on $\partial N$ whose leaves are closed curves. There exists a non-fuchsian geometrically finite metric $g$ such that $\alpha$ is the measured pleating lamination of the boundary of the convex core if and only if the following conditions are satisfied: 
(1) Each leaf of $\alpha$ has weight at most $\pi$.

(2) For all essential annulus or Möbius strip $A$ in $N$, the total weight of $\partial A$ is strictly positive.

(3) For all essential disk $D$ in $N$, the total weight of $\partial D$ is strictly larger than $2 \pi$.

This metric $g$ is then unique (up to isotopy in $N$ ).

The proof of this difficult result uses among other things a result of Hodgson and Kerckhoff [HK98] on the rigidity of hyperbolic cone-manifolds.

3.3. Proof of Theorem 2.4 from Theorem 3.1. First note that condition (1) of Theorem 2.4, that the cellular decomposition of $\partial^{\prime} N$ is proper, is necessary. If it were not satisfied, and if $M$ were a manifold with hyperideal boundary as described in the statement of Theorem 2.4, the boundary of the universal cover $\tilde{M}$ of $M$, considered as a complete convex polyhedral surface in $H^{3}$, would contain a non-trivial closed path contained in the union of the closures of two faces. This is clearly impossible by convexity.

The rest of the proof proceeds in several steps.

1. The uniqueness in Theorem 2.4. Let $N, \Gamma$ and $w$ be as in the statement of Theorem 2.4. Suppose first that, as stated in the theorem, there exists a strictly hyperideal hyperbolic metric $g$ on $N$ such that the boundary combinatorics of $N$ is given by $\Gamma$ and its dihedral angles by $w$. Let $N_{t}$ be the truncated version of $N$. So $N_{t}$ is a finite volume hyperbolic manifold with convex, polyhedral boundary; its boundary has one face for each face of $\Gamma$, and one - induced by the truncation - for each vertex of $\Gamma$ (i.e. for each hyperideal vertex of $N$ ). Moreover, each face corresponding to a vertex of $\Gamma$ is orthogonal to each face corresponding to an adjacent face of $\Gamma$.

Consider another copy of $N_{t}$, which we call $N_{t}^{\prime}$, and glue $N_{t}$ to $N_{t}^{\prime}$ along all the couples of faces - one on $N_{t}$ and the other on $N_{t}^{\prime}$ - corresponding to the vertices of $\Gamma$; let $C$ be the resulting hyperbolic manifold. By construction, $C$ has finite volume - since $N_{t}$ and $N_{t}^{\prime}$ have finite volume - and it has a "polyhedral" boundary, with no vertex.

Each face $f$ of $\Gamma$ has two corresponding faces, one, say $F$, on $\partial N_{t}$, and the other, say $F^{\prime}$, on $\partial N_{t}^{\prime}$. But, for each vertex $v$ of $\Gamma$ adjacent to $f$, the faces on $\partial N_{t}$ and $\partial N_{t}^{\prime}$ corresponding to $v$ are orthogonal to $F$ and $F^{\prime}$, respectively. If follows that $F$ and $F^{\prime}$ are two parts of the same face of $C$. Each edge of $F$ with, on the other side, a face of $\partial N_{t}$ corresponding to a vertex of $\Gamma$ "disappears" in $C$, while each edge of $F$ with on the other side a face of $\partial N_{t}$ contained in a face of $\partial N$ becomes, in $C$, half of an edge of $\partial C$ which is a closed curve.

The following description of $\partial C$ follows from those considerations. It has:

- One face $F_{c}$ for each face $f$ of $\Gamma$, and $F_{c}$ is topologically a sphere with $v$ disks removed, where $v$ is the number of vertices of $f$.

- One edge for each edge of $\Gamma$, and each of those edges is a closed curve. The (exterior) dihedral angle at each edge is equal to the value of $w$ on the corresponding edge of $\Gamma$. 
It cleary follows from this description that $C$ is the convex core of a geometrically finite hyperbolic 3-manifolds, which has a rational pleating lamination. The uniqueness in Theorem 3.1 shows that $C$ is uniquely determined by $\Gamma$ and by $w$, and the uniqueness in Theorem 2.4 follows.

2. Conditions (2) and (3) of Theorem 2.4 are necessary. For condition (2), consider a closed curve $\gamma$ as in the statement of condition (2), so that $\gamma$ is a sequence of faces of $\partial M$, with two consecutive faces sharing an edge. Associate to $\gamma$ a curve $\gamma^{\prime}$ in $\partial M$, which intersects the same faces in the same order. Following the construction made in step (1), $\gamma^{\prime}$ can be considered as a closed curve in the boundary of $C$. Since $\gamma$ is simple, this curve is homotopically non-trivial in $\partial C$, while it is homotopically trivial in $C$. But $\partial C$ lifts to a complete, convex, polyhedral surface in $H^{3}$. It is known that, for any non-trivial closed curve on such a surface, the sum of the exterior dihedral angles of the edges crossed by the closed curve is strictly larger than $2 \pi$, see [RH93, CD95, Sch96, Sch98]. Condition (2) follows.

For condition (3), consider an open curve $c$ as in the statement of condition (3). In the manifold $C$ constructed in step (1) above, consider two copies of $c$, one on $\partial N_{t}$, and the other on $\partial N_{t}^{\prime}$; they can be glued at their endpoints to obtain a closed, non-trivial curve in $C$, with boundary in $\partial C$. The same argument as for condition (2) thus shows that the sum of the exterior dihedral angles of the edges of $\partial C$ crossed by this closed path has to be strictly larger than $2 \pi$, and the statement of condition (3) follows.

3. Construction of a manifold with a rational measured lamination. To prove the existence of $M$, we consider a 3 -manifold $\mathcal{C}$ along with a rational measured lamination $\alpha$ on $\partial \mathcal{C}$ corresponding to the manifold $C$ obtained above. So $\mathcal{C}$ is obtained by gluing two copies of $N$, which we call $N$ and $N^{\prime}$, along a set of disks, one for each vertex of $\Gamma$. Since the interior of $N$ has a complete hyperbolic metric, $N$ is irreducible and homotopically atoroidal; since $\mathcal{C}$ is obtained by gluing two copies of $N$ along some disks in the boundaries, it follows that $\mathcal{C}$ is also irreducible and homotopically atoroidal (and $\mathcal{C}$ is not the interval bundle over the Klein bottle), so that $\mathcal{C}$ admits a complete hyperbolic metric.

The lamination $\alpha$ has one closed curve for each edge of $\Gamma$, so that is has a finite number of closed leaves. The transverse measure we consider is the one given, for each leaf of $\alpha$, by the value of $w$ on the corresponding edge of $\Gamma$. Clearly hypothesis (1) of Theorem 3.1 is satisfied.

4. Proof that hypothesis (3) of Theorem 3.1 is satisfied. Let $D$ be an essential disk in $\mathcal{C}$. We can suppose (applying a small deformation if necessary) that its boundary $\partial D \subset \partial \mathcal{C}$ is transverse to the edges of $\partial \mathcal{C}$. In addition, it is possible to make two kinds of deformations of $D$ to obtain a simpler picture.

- Suppose that some connected component $D^{\prime}$ of $D \cap N$ is a (closed) disk in the interior of $D$. Then $\partial D^{\prime}$ is contained in one of the disks along which $N$ is glued to $N^{\prime}$. Since $N$ is irreducible, $D^{\prime}$ can be deformed to the disk in $N \cap N^{\prime}$ bounded by $\partial D^{\prime}$. It is then possible to "push" a 
neighborhood of $D^{\prime}$ in $D$ into $N^{\prime}$. The same argument can be applied to a connected component $D^{\prime}$ of $D \cap N^{\prime}$ under the same hypothesis.

- Suppose that some connected component $D^{\prime}$ of $D \cap N$ is such that $\partial D^{\prime}=$ $c \cup c^{\prime}$, where $c \subset \partial D \cap \partial N$ and $c^{\prime} \subset N \cap N^{\prime}$ are connected curves. Suppose moreover that $c$ remains in the union of the faces of $\partial N$ which are adjacent to the disk of $N \cap N^{\prime}$ containing $c^{\prime}$. Then the same argument shows that a neighborhood of $D^{\prime}$ in $D$ can be "pushed" into $N^{\prime}$. Again the same argument works if $D^{\prime} \subset N^{\prime}$.

Finally, perturbing $D$ a little if necessary, we suppose that $D$ is transverse to the surface $N \cap N^{\prime}$ between $N$ and $N^{\prime}$.

We associate to $\partial D$ a closed path $\gamma$ in the dual graph $\Gamma^{*}$ of $\Gamma$, which follows the edges dual to the edges crossed by $\partial D$. The total weight of $\partial D$ for the transverse measure $\alpha$ is equal to the sum of the values of $w$ on the edges of $\gamma$. If $D \subset N$ (or if $D \subset N^{\prime}$ ) then hypothesis (3) of Theorem 3.1 is a direct translation of hypothesis (1) of Theorem 2.4. We now suppose that it is not the case.

Consider the connected components $D_{1}, \cdots, D_{N}$ of either $(D \cap N)$ or $\left(D \cap N^{\prime}\right)$. By the simplifications done above, each of the $D_{i}$ has non-empty intersection with $\partial D$. Since $D$ is a disk, at least two of those connected components, say $D_{1}$ and $D_{2}$, have a boundary which is the union of one (connected) curve of $\partial D$ and of one connected component of $D \cap N \cap N^{\prime}$. Then the curve $\partial D_{1} \cap \partial D$ (resp. $\partial D_{2} \cap \partial D$ ) is in $\partial N \cap \partial \mathcal{C}$ (or in $\partial N^{\prime} \cap \partial \mathcal{C}$ ) and begins and ends on a truncation face $f_{1}$ (resp. $f_{2}$ ). Moreover, the deformation made above shows that $\partial D_{1} \cap \partial D$ (resp. $\partial D_{2} \cap \partial D$ ) does not remain in the faces adjacent to $f_{1}$ (resp. $f_{2}$ ). Therefore hypothesis $(2)$ of Theorem 2.4 shows that its total weight for $\alpha$ is strictly larger than $\pi$. It follows that the total weight of $\partial D$ for $\alpha$ is strictly larger than $2 \pi$, so that hypothesis (3) of Theorem 3.1 is valid.

5. Proof that hypothesis (2) of Theorem 3.1 is satisfied. Let $A$ be an essential annulus in $\mathcal{C}$, such that the total weight of $\partial A$ for $\alpha$ is zero; then each connected component of $\partial A$ is contained in one of the faces of $\mathcal{C}$. It follows that each of the connected components of $\partial A$ enters both $N$ and $N^{\prime}$, otherwise it would be a closed curve in a convex cell, and $A$ could not be essential.

We can suppose that $A$ is transverse to $N \cap N^{\prime}$. Note that no connected component of $A \cap\left(N \cap N^{\prime}\right)$ is a closed non-trivial loop in the interior of $A$, because otherwise - since $N \cap N^{\prime}$ is the disjoint union of a finite number of disks - $A$ could not be an essential annulus.

Moreover, if some connected component of $A \cap\left(N \cap N^{\prime}\right)$ is a homotopically trivial loops in $A$, we can suppress it by deforming $A$, as described for disks in the previous step. So we can suppose that all the connected components of $A \cap\left(N \cap N^{\prime}\right)$ intersect $\partial A$. The same deformation argument can be applied if one connected component of $A \cap\left(N \cap N^{\prime}\right)$ has both endpoints on the same connected component of $\partial A$. So we can suppose that all connected components of $A \cap\left(N \cap N^{\prime}\right)$ go from one connected component of $\partial A$ to the other. 
Let $D$ be one of the connected components of either $A \cap N$ or $A \cap N^{\prime}$. Then $D$ is a strip in $A$, bounded by two curves, each going from one of the connected components of $\partial A$ to the other. So $D$ is a disk in $N$ (or in $N^{\prime}$ ), with boundary in $\partial N\left(\right.$ or $\left.\partial N^{\prime}\right)$ a curve which intersects the closure of at most two cells of $\Gamma$ in $\partial N$ (or $\partial N^{\prime}$ ) corresponding to the faces of $\mathcal{C}$ containing the two boundary curves of $A$. If $D$ is essential, this is impossible since it contradicts the hypothesis that the cellular decomposition of $\partial^{\prime} N$ is proper. However, if none of the connected component of $A \cap N$ or $A \cap N^{\prime}$ is essential, $A$ is not essential. The same argument can be used if $A$ is a Mbius strip, and this shows that hypothesis (2) in Theorem 3.1 is valid.

6. End of the proof. Theorem 3.1 shows that there exists a unique hyperbolic metric $h$ on $\mathcal{C}$ such that $(\mathcal{C}, h)$ is the convex core of a geometrically finite manifold, with rational pleating lamination, with pleating lamination given by $\Gamma$ and transverse measure given by $\alpha$. By the uniqueness, $(\mathcal{C}, h)$ can be cut along totally geodesic surfaces to obtain hyperbolic metrics on $N$ and $N^{\prime}$, corresponding to truncated hyperideal metrics. Theorem 2.4 follows.

\section{Acknowledgements}

The results presented here owe a lot to a conversation with Francis Bonahon. I'm also grateful to Boris Springborn for some conversations directly related to this paper.

\section{References}

[And71] E. M. Andreev, On convex polyhedra of finite volume in Lobacevskii space. Math. USSR Sbornik, 12 (1971), 225-259.

[BB02] X. Bao, F. Bonahon, Hyperideal polyhedra in hyperbolic 3-space, Bull. Soc. Math. France, 130 (2002), 457-491.

[BO01] F. Bonahon and J.-P. Otal, Laminations mesurées de plissage des variétés hyperboliques de dimension 3, http://math.usc.edu/ fbonahon; Ann. Math., to appear., 2001.

[Bon01] F. Bonahon, Geodesic laminations on surfaces, In Laminations and foliations in dynamics, geometry and topology (Stony Brook, NY, 1998), 269 of Contemp. Math., pages 1-37. Amer. Math. Soc., Providence, RI, 2001.

[BS04] A. I. Bobenko, B. A. Springborn, Variational principles for circle patterns and Koebe's theorem, Trans. Amer. Math. Soc., 356 (2004), 659-689 (electronic).

[CD95] R. Charney and M. Davis, The polar dual of a convex polyhedral set in hyperbolic space, Michigan Math. J., 42 (1995), 479-510.

[EM86] D. B. A. Epstein and A. Marden, Convex hulls in hyperbolic spaces, a theorem of Sullivan, and measured pleated surfaces, In D. B. A. Epstein, editor, Analytical and geometric aspects of hyperbolic space, 111 of L.M.S. Lecture Note Series. Cambridge University Press, 1986.

[GW93] P. M. Gruber and J. M. Wills, editors, Handbook of convex geometry. Vol. A, B. North-Holland Publishing Co., Amsterdam, 1993.

[HK98] C. D. Hodgson and S. P. Kerckhoff, Rigidity of hyperbolic cone-manifolds and hyperbolic Dehn surgery, J. Differential Geom., 48 (1998), 1-60.

[Koe36] P. Koebe, Kontaktprobleme der konformen Abbildung, Abh. Sächs. Akad. Wiss. Leipzig Math.-Natur. Kl., 88 (1936), 141-164. 
[Lei02] G. Leibon, Characterizing the Delaunay decompositions of compact hyperbolic surfaces, Geom. Topol., 6 (2002), 361-391 (electronic).

[Ota94] J.-P. Otal, Sur le coeur convexe d'une variété hyperbolique de dimension 3, Preprint, 1994.

[RH93] I. Rivin and C. D. Hodgson, A characterization of compact convex polyhedra in hyperbolic 3-space, Invent. Math., 111 (1993), 77-111.

[Riv86] I. Rivin, Thesis. PhD thesis, Princeton University, 1986.

[Riv96] _ A characterization of ideal polyhedra in hyperbolic 3-space, Annals of Math., 143 (1996), 51-70.

[Riv03] C Combinatorial optimization in geometry, Adv. in Appl. Math., 31 (2003), $242-271$

[Rou04] M. Rousset, Sur la rigidité de polyèdres hyperboliques en dimension 3 : cas de volume fini, cas hyperidéal, cas fuchsien, Bull. Soc. Math. France, 132 (2004), 233-261.

[Sch96] J.-M. Schlenker, Surfaces convexes dans des espaces lorentziens à courbure constante, Commun. Anal. and Geom., 4 (1996), 285-331.

[Sch98] _ Métriques sur les polyèdres hyperboliques convexes, J. Differential Geom., 48 (1998), 323-405.

[Sch02] Hyperideal polyhedra in hyperbolic manifolds, Preprint math.GT/0212355., 2002.

[Thu80] W. P. Thurston, Three-dimensional geometry and topology, Recent version available on http://www.msri.org/publications/books/gt3m/, 1980.

[Thu82] _ , Three-dimensional manifolds, Kleinian groups and hyperbolic geometry, Bull. Amer. Math. Soc. (N.S.), 6 (1982), 357-381.

Laboratoire Emile Picard, UMR CNRS 5580, Université Paul Sabatier, 118 route de Narbonne, 31062 Toulouse Cedex 4, France.

E-mail address: schlenker@picard.ups-tlse.fr

$U R L:$ http://picard.ups-tlse.fr/ schlenker 